\title{
Thermocapillary migration of a droplet with a thermal source at large Reynolds and Marangoni numbers
}

\author{
Zuo-Bing $\mathrm{Wu}^{*}$ \\ State Key Laboratory of Nonlinear Mechanics, Institute of Mechanics, Chinese Academy of Sciences, Beijing 100190, China
}

\section{A R T I C L E I N F O}

\section{Article history:}

Received 31 October 2013

Received in revised form 1 April 2014

Accepted 5 April 2014

\section{Keywords:}

Interfacial tension

Thermocapillary droplet migration

Large Marangoni number

Microgravity

\begin{abstract}
A B S T R A C T
The unsteady process for thermocapillary droplet migration at large Reynolds and Marangoni numbers has been previously reported by identifying a nonconservative integral thermal flux across the surface in the steady thermocapillary droplet migration ( $\mathrm{Wu}$ and $\mathrm{Hu}, 2013)$ [15]. Here we add a thermal source in the droplet to preserve the integral thermal flux across the surface as conservative, so that thermocapillary droplet migration at large Reynolds and Marangoni numbers can reach a quasi-steady process. Under assumptions of quasi-steady state and non-deformation of the droplet, we make an analytical result for the steady thermocapillary migration of droplet with the thermal source at large Reynolds and Marangoni numbers. The result shows that the thermocapillary droplet migration speed slowly increases with the increase of Marangoni number.
\end{abstract}

(c) 2014 Elsevier Ltd. All rights reserved.

\section{Introduction}

Thermocapillary migration of a droplet or bubble at a uniform temperature gradient is a very interesting topic in both microgravity and microfluidics[1,2]. On the one hand, due to the development of space exploration, the studies of the physical mechanism of the droplet/bubble migration phenomena in the microgravity environment become more and more important. The first investigation of thermocapillary droplet migration was completed by Young et al (YGB) in 1959, who gave an analytical prediction for the migration speed at zero limit Reynolds (Re) and Marangoni (Ma) numbers [3]. Subramanian [4] proposed the quasi-steady state assumption for thermocapillary bubble migration and obtained analytical results with high order expansion at small Ma numbers. Balasubramaniam and Chai [5] derived an exact result for thermocapillary droplet migration at small Ma numbers. The experimental results for the droplet migration speed at small Re numbers obtained by Braun et al [6] are in agreement with those of the YGB model. On the other hand, the manipulation and actuation of droplets in microfluidic devices have an extensive application on the chemical industry and biological engineering. Many interest problems, such as static contact and dynamic control of the droplets on solid substrates, were theoretically analyzed and experimentally observed [7-10].

\footnotetext{
* Tel.: +86 10 82543955; fax: +86 1082543977 .

E-mail address: wuzb@lnm.imech.ac.cn
}

Although the thermocapillary droplet migration processes at small Ma numbers under the microgravity environment are understood very well in the series of theoretical analyses and experimental investigations, the physical behaviors at large Ma numbers are rather complicated due to the momentum and energy transfer though the interface of two-phase fluids. In large Re and Ma number area, it was reported [11] that the migration speed of a droplet increases with the increase of Ma number, as is in qualitative agreement with the corresponding numerical simulation[12]. The above theoretical analysis and numerical simulation are based on assumptions of the quasi-steady state and non-deformation of the droplet. The experimental investigation carried out by Hadland et al. [13] and Xie et al. [14] showed that the droplet migration speed non-denationalized by the YGB velocity decreases with the increase of Ma number. The results are not in qualitative agreement with the above theoretical and numerical ones. Moreover, a nonconservative integral thermal flux across the surface in the steady thermocapillary droplet migration at large $\mathrm{Ma}(\mathrm{Re})$ numbers was identified [15]. It was indicated that the thermocapillary droplet migration at large $\mathrm{Ma}(\mathrm{Re})$ numbers is an unsteady process. Therefore, the thermocapillary droplet migration at large Ma (Re) numbers remains a topic to be studied with respect to its physical mechanism.

In this paper, first, under the assumption of the quasi-steady process of thermocapillary droplet migration at large Re and Ma numbers, a thermal source is added in the droplet to change the integral thermal flux across the surface from nonconservative to conservative. Then, we make an analytical result for the steady 
thermocapillary migration of droplet with the thermal source at large Re and Ma numbers and elucidate effects of the adding thermal source in the droplet on thermocapillary migration process.

\section{Problem formulation}

Consider the thermocapillary migration of a spherical droplet of radius $R_{0}$, density $\gamma \rho$, dynamic viscosity $\alpha \mu$, thermal conductivity $\beta k$, and thermal diffusivity $\lambda \kappa$ in a continuous phase fluid of infinite extent with density $\rho$, dynamic viscosity $\mu$, thermal conductivity $k$, and thermal diffusivity $\kappa$ under a uniform temperature gradient $G$. The rate of change of the interfacial tension between the droplet and the continuous phase fluid with temperature is denoted by $\sigma_{T}$. Fig. 1 displays a schematic diagram of thermocapillary migration of a droplet with a thermal source in a laboratory coordinate system denoted by a bar. Axisymmetric energy equations for the continuous phase and the fluid within the droplet are written as follows

$$
\begin{aligned}
& \frac{\partial \bar{T}}{\partial t}+\overline{\mathbf{v}} \bar{\nabla} \bar{T}=\kappa \bar{\Delta} \bar{T}, \\
& \frac{\partial \bar{T}^{\prime}}{\partial t}+\overline{\mathbf{v}}^{\prime} \bar{\nabla} \bar{T}^{\prime}=\lambda \kappa \bar{\Delta} \bar{T}^{\prime}+\Omega,
\end{aligned}
$$

where $\overline{\mathbf{v}}$ and $\bar{T}$ are velocity and temperature, a prime and $\Omega$ denote quantities and a thermal source in the droplet. By going over an unstable migration process from beginning, the droplet migration can reach a steady state, i.e., with the constant migration speed $V_{\infty}$. Using the coordinate transformation from the laboratory coordinate system to a coordinate system moving with the droplet velocity $V_{\infty}$

$$
\begin{aligned}
& \overline{\mathbf{r}}=\mathbf{r}+V_{\infty} t \mathbf{k}, \quad \overline{\mathbf{v}}(\overline{\mathbf{r}}, t)=\mathbf{v}(\mathbf{r})+V_{\infty} \mathbf{k}, \quad \bar{T}(\overline{\mathbf{r}}, t)=T(\mathbf{r})+G V_{\infty} t, \\
& \overline{\mathbf{v}}^{\prime}(\overline{\mathbf{r}}, t)=\mathbf{v}^{\prime}(\mathbf{r})+V_{\infty} \mathbf{k}, \quad \bar{T}^{\prime}(\overline{\mathbf{r}}, t)=T^{\prime}(\mathbf{r})+G V_{\infty} t,
\end{aligned}
$$

the problem (1) can be formulated as

$$
\begin{aligned}
& G V_{\infty}+\mathbf{v} \nabla T=\kappa \Delta T, \\
& G V_{\infty}+\mathbf{v}^{\prime} \nabla T^{\prime}=\lambda \kappa \Delta T \prime+\Omega .
\end{aligned}
$$

By taking the radius of the droplet $R_{0}$, the YGB model velocity $v_{0}=-\sigma_{T} G R_{0} / \mu$ and $G R_{0}$ as reference quantities to make coordinates, velocity and temperature dimensionless, the energy Eq. (3) can be written in the following dimensionless form in the spherical coordinate system $(r, \theta)$ as follows

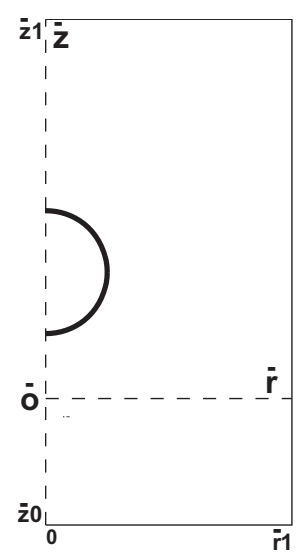

Fig. 1. A schematic diagram of thermocapillary migration of droplet with a thermal source in a laboratory coordinate system.

$$
\begin{aligned}
& 1+u \frac{\partial T}{\partial r}+\frac{v}{r} \frac{\partial T}{\partial \theta}=\epsilon^{2} \Delta T, \\
& 1+u^{\prime} \frac{\partial T^{\prime}}{\partial r}+\frac{v^{\prime}}{r} \frac{\partial T^{\prime}}{\partial \theta}=\lambda \epsilon^{2} \Delta T^{\prime}+\frac{\Omega}{G v_{0} V_{\infty}},
\end{aligned}
$$

where the small parameter $\epsilon$ and Ma number are defined, respectively, as

$\epsilon=\frac{1}{\sqrt{\operatorname{MaV}_{\infty}}}$

and

$M a=\frac{v_{0} R_{0}}{\kappa}$.

In the space experiments[13,14], Prandtl numbers $\left(\operatorname{Pr}=\frac{\mu}{\rho \kappa}\right)$ are constants in terms of the fixed fluid media, but Ma numbers are changed duo to the variable droplet sizes. It is common to adopt Re numbers $\left(R e=\frac{\rho v_{0} R_{0}}{\mu}=\frac{M a}{P r}\right)$ instead of Pr numbers in order to capture velocity fields with the changes of Re numbers. In general, the deformation of droplet usually depends on the Weber numbers $\left[W e=\frac{\rho v_{0}^{2} R}{\sigma_{0}}=\operatorname{CaRe}, \mathrm{Ca}\left(=\frac{v_{0} \mu}{\sigma_{0}}\right)\right.$ is the Capillary number], it is not included in this study due to the small We numbers in the space experiments. For example, $W e$ is in $O\left(10^{-3}\right)-O\left(10^{-1}\right)$ when $M a<1000[14,16]$. Thus, the solutions of Eqs. (4) and (5) have to satisfy the following boundary conditions at the interface of two phase fluids $(r=1)$

$T(1, \theta)=T^{\prime}(1, \theta)$,

$\frac{\partial T}{\partial r}(1, \theta)=\beta \frac{\partial T^{\prime}}{\partial r}(1, \theta)$

and at places far away from the droplet

$T \rightarrow r \cos \theta, \quad$ as $r \rightarrow \infty$.

Besides the above temperature boundary conditions, the stress boundary conditions at the interface are also applied for the steady droplet migration. However, the normal stress boundary condition is removed in terms of the non-deformation assumption. The shear stress boundary condition is expressed by

$\tau_{r \theta}-\alpha \tau_{r \theta}^{\prime}=\frac{1}{V_{\infty} r} \frac{\partial T}{\partial \theta}$,

where

$\tau_{r \theta}=r \frac{\partial}{\partial r}\left(\frac{v}{r}\right)+\frac{1}{r} \frac{\partial u}{\partial \theta}$.

For large Re numbers, the inner and outer momentum boundary layers at $r$-direction are introduced near the surface of droplet. In the flow fields outside the momentum boundary layers, there are still the potential flows. The scaled inviscid velocity field in the continuous phase and Hill's spherical vortex within the droplet can be written as, respectively [17]

$U=-\cos \theta\left(1-\frac{1}{r^{3}}\right)$,
$V=\sin \theta\left(1+\frac{1}{2 r^{3}}\right)$

and

$U^{\prime}=\frac{3}{2} \cos \theta\left(1-r^{2}\right)$,

$V^{\prime}=-\frac{3}{2} \sin \theta\left(1-2 r^{2}\right)$.

By introducing the perturbation velocity fields $\hat{\mathbf{v}}=\mathbf{v}-\mathbf{U}$, $\hat{\mathbf{v}}^{\prime}=\mathbf{v}^{\prime}-\mathbf{U}^{\prime}$ and the depth of boundary layer $\delta x=r-1\left(\delta=\sqrt{\frac{1}{R e}}\right)$ 
into the boundary condition (11) and the momentum equation for the continuous fluid, they are derived and truncated in the leading orders $O(1 / \delta)$ and $O(\delta)$ as, respectively [17]

$\frac{\partial \hat{v}}{\partial x}=\frac{3}{2}(2+3 \alpha) \sin \theta+\frac{1}{V_{\infty}} \frac{\partial T}{\partial \theta}$.

and

$\frac{3}{2} \frac{\partial}{\partial \theta}(\hat{v} \sin \theta)-3 x \cos \theta \frac{\partial \hat{v}}{\partial x}=\frac{\partial^{2} \hat{v}}{\partial x^{2}}$,

where $\frac{\partial}{\partial r}=O\left(\frac{1}{\delta}\right), U / U^{\prime}=O(\delta) / O(\delta), V / V^{\prime}=O(1) / O(1), \hat{u} / \hat{u}^{\prime}=O\left(\delta^{2}\right) /$ $O\left(\delta^{2}\right)$ and $\hat{v} / \hat{v}^{\prime}=O(\delta) / O(\delta)$ are in the momentum boundary layers. Following the derivation for the steady migration speed of a bubble at large Re numbers [18], the migration speed of the droplet is written as follows

$$
\begin{aligned}
V_{\infty} & =-\frac{1}{2(2+3 \alpha)} \int_{0}^{\pi} \sin ^{2} \theta \frac{\partial T}{\partial \theta}(1, \theta) d \theta \\
& =\frac{1}{2+3 \alpha} \int_{0}^{\pi} \sin \theta \cos \theta T(1, \theta) d \theta .
\end{aligned}
$$

Thus, to determine $V_{\infty}$, the temperature $T(1, \theta)$ on the surface of droplet is required. However, before the energy equations are solved, the self-consistency of temperature fields for the steady droplet migration system will be determined. It means that the solutions of steady energy equations will satisfy the conservative integral thermal flux across the surface of droplet. In this case, the temperature field at infinity in Eq. (10) was derived by using the asymptotic expansion method and expressed as [15]

$T \approx r \cos \theta-\frac{1}{2 r^{2}} \cos \theta+o(1)$.

Integrating Eqs. (4) and (5) in the continuous phase domain $\left(r \in\left[1, r_{\infty}\right], \theta \in[0, \pi]\right)$ with boundary condition (18) and within the droplet region $(r \in[0,1], \theta \in[0, \pi])$, respectively, we obtain

$\int_{0}^{\pi} \frac{\partial T}{\partial r}(1, \theta) \sin \theta d \theta=-\frac{1}{3 \epsilon^{2}}$

and

$\int_{0}^{\pi} \frac{\partial T^{\prime}}{\partial r}(1, \theta) \sin \theta d \theta=\frac{2}{3 \lambda \epsilon^{2}}\left(1-\frac{\Omega}{G v_{0} V_{\infty}}\right)$.

From Eqs. (19) and (20), we have

$$
\begin{aligned}
\beta & \int_{0}^{\pi} \frac{\partial T^{\prime}}{\partial r}(1, \theta) \sin \theta d \theta-\int_{0}^{\pi} \frac{\partial T}{\partial r}(1, \theta) \sin \theta d \theta \\
& =\int_{0}^{\pi}\left[\beta \frac{\partial T^{\prime}}{\partial r}(1, \theta)-\frac{\partial T}{\partial r}(1, \theta)\right] \sin \theta d \theta \\
& =\frac{1}{3 \epsilon^{2}}\left(1+\frac{2 \beta}{\lambda}\right)-\frac{2 \beta \Omega}{3 \lambda G v_{0}} M a
\end{aligned}
$$

For large Ma numbers and finite $V_{\infty}$, Eqs. (19) and (20) should satisfy the thermal flux boundary condition (9), i.e., the right side of Eq. (21) will be zero. So, we have

$\Omega=\frac{\lambda G v_{0} V_{\infty}}{2 \beta}\left(1+\frac{2 \beta}{\lambda}\right)=G v_{0} V_{\infty}\left(1+\frac{\lambda}{2 \beta}\right)$

Using Eq. (22), Eq.(5) is rewritten as

$u^{\prime} \frac{\partial T^{\prime}}{\partial r}+\frac{v^{\prime}}{r} \frac{\partial T^{\prime}}{\partial \theta}=\lambda \epsilon^{2} \Delta T^{\prime}+\frac{\lambda}{2 \beta}$.

In following, we will focus on the steady thermocapillary migration of droplet with the thermal source under a uniform temperature gradient and determine the dependence of the migration speed on large Ma numbers.

\section{Analysis and results}

\subsection{Outer temperature field in the continuous phase}

By using an outer expansion for the scaled temperature field in the continuous phase

$T=T_{0}+T_{1} \epsilon+O\left(\epsilon^{2}\right)$,

the energy equation for the outer temperature field in its leading order can be obtained from Eq. (4) as follows

$1+U \frac{\partial T_{0}}{\partial r}+\frac{V}{r} \frac{\partial T_{0}}{\partial \theta}=0$

By using the coordinate transformation from $(r, \theta)$ to $\left(\psi_{0}, \theta\right)$ to solve Eq. (25), its solution can be written as

$T_{0}(r, \theta)=G\left(\psi_{0}\right)-\int \frac{2 r^{4}}{2 r^{3}+1} \frac{d \theta}{\sin \theta}$,

where $G\left(\psi_{0}\right)$ is a function of $\psi_{0}$ (the streamfunction in the continuous phase). Following[19], the solution near $r=1$ is simplified as

$$
\begin{aligned}
T_{0}(r, \theta)= & \left(1+\frac{\pi}{6 \sqrt{3}}-\frac{1}{6} \ln 432\right) \\
& -\frac{1}{18}\left(\frac{\pi}{\sqrt{3}}+\ln 432\right)\left(r^{2}-\frac{1}{r}\right) \sin ^{2} \theta+\frac{1}{3} \\
& \times \ln \left(r^{2}-\frac{1}{r}\right)+\frac{2}{3} \ln (1+\cos \theta)+\frac{2}{9}\left(r^{2}-\frac{1}{r}\right) \cos \theta \\
& +\frac{1}{9}\left(r^{2}-\frac{1}{r}\right) \ln \left(r^{2}-\frac{1}{r}\right) \sin ^{2} \theta \\
& +\frac{2}{9}\left(r^{2}-\frac{1}{r}\right) \sin ^{2} \theta \ln (1+\cos \theta) .
\end{aligned}
$$

By using the boundary layer approximation

$x=\frac{r-1}{\epsilon}$,

the temperature field near interface can be expressed as

$$
\begin{aligned}
t(x, \theta)= & 1+\frac{\pi}{6 \sqrt{3}}-\frac{1}{6} \ln 48+\frac{2}{3} \ln \left(\frac{1+\cos \theta}{\sin \theta}\right) \\
& +\frac{1}{3} x \sin ^{2} \theta \epsilon \ln \epsilon+O(\epsilon) .
\end{aligned}
$$

\subsection{Outer temperature field within the droplet}

By using the following outer expansion for the scaled temperature field within the droplet in Eq. (23)

$T^{\prime}=\frac{1}{\epsilon^{2}} T_{-2}^{\prime}+T_{0}^{\prime}+O(\epsilon)$,

the equation in its leading order can be written as

$U^{\prime} \frac{\partial T_{-2}^{\prime}}{\partial r}+\frac{V^{\prime}}{r} \frac{\partial T_{-2}^{\prime}}{\partial \theta}=0$

Its solution is

$T_{-2}^{\prime}=F_{0}\left(\psi^{\prime}\right)$,

where $\psi^{\prime}=\frac{3}{4} \sin ^{2} \theta\left(r^{4}-r^{2}\right)$ is the streamfunction within the droplet. The unknown function $F_{0}\left(\psi^{\prime}\right)$ can be obtained from the following equation for the temperature field $T_{0}^{\prime}$ in its second order

$U^{\prime} \frac{\partial T_{0}^{\prime}}{\partial r}+\frac{V^{\prime}}{r} \frac{\partial T_{0}^{\prime}}{\partial \theta}=\lambda \Delta F_{0}+\frac{\lambda}{2 \beta}$.

To solve Eq. (33), we use the coordinate transformation from $(r, \theta)$ to $(m, q)$, with streamlines and their orthogonal lines as coordinate axes defined as 
$m=-\frac{16}{3} \psi^{\prime}$,

$q=\frac{r^{4} \cos ^{4} \theta}{2 r^{2}-1}$

Eq. (33) can thus be written in the $(m, q)$ coordinate system as follows

$\frac{V_{q}}{h_{q}} \frac{\partial T_{0}^{\prime}}{\partial q}=\frac{\lambda}{h_{m} h_{q} h_{\phi}} \frac{\partial}{\partial m}\left(\frac{h_{q} h_{\phi}}{h_{m}} \frac{d F_{0}}{d m}\right)+\frac{\lambda}{2 \beta}$,

where $h_{m}, h_{q}, h_{\phi}$ are the metrical coefficients for the transformation. Multiplying both sides of Eq. (35) by $h_{m} h_{q} h_{\phi}$ and integrating with respect to $q$ for one circuit along a streamline, we obtain the following equation

$\oint V_{q} h_{m} h_{\phi} \frac{\partial T_{0}^{\prime}}{\partial q} d q=\lambda \frac{\partial}{\partial m}\left[J(m) \frac{d F_{0}}{d m}\right]+\frac{\lambda}{2 \beta} H(m)$,

where $H(m)=\oint h_{m} h_{q} h_{\phi} d q$ and $J(m)=\oint \frac{h_{q} h_{\phi}}{h_{m}} d q$. From the equation of continuity, it can be shown that $V_{q} h_{m} h_{\phi}$ is a constant. We thus obtain a solution of Eq. (36) as follows

$F_{0}=-\frac{1}{2 \beta}\left[B+\int_{0}^{m} \frac{d x}{J(x)} \int_{1}^{x} H(s) d s\right]$.

Near $m=0, J(m)$ and $H(m)$ can be expanded, respectively

$J(m)=\frac{16}{3}-5 m+O\left(m^{2} \ln m\right)$

and

$H(m)=\frac{3}{8} \ln 2-\frac{1}{16} \ln m+O(m \ln m)$.

Using Eqs. (38) and (39), we thus obtain from Eq. (37)

$$
\begin{aligned}
T_{-2}^{\prime}(r, \theta)= & F_{0} \\
= & -\frac{1}{2 \beta}\left[B-\frac{1}{16} m+\frac{3}{256}\left(3 \ln 2-1 \frac{3}{4}\right) m^{2}-\frac{3}{512} m^{2} \ln m\right] \\
& +O\left(m^{3} \ln m\right) .
\end{aligned}
$$

By using the boundary layer approximation

$x^{\prime}=\frac{1-r}{\sqrt{\lambda} \epsilon}$,

the temperature field near interface can be expressed as follows

$t^{\prime}\left(x^{\prime}, \theta\right)=\frac{\sqrt{\lambda}}{4 \beta} x^{\prime} \sin ^{2} \theta \frac{1}{\epsilon}+\frac{3 \lambda}{16 \beta} x^{\prime 2} \sin ^{4} \theta \ln \epsilon+o(\ln \epsilon)$.

\subsection{Inner temperature fields in the leading order}

From Eqs. (19) and (20), the leading orders of the inner temperature fields in the continuous phase and within the droplet can be obtained, respectively. A schematic diagram of the inner and outer regions with the leading orders near the surface of droplet is shown in Fig. 2. By using inner expansions for the continuous phase and the fluid in the droplet

$t(x, \theta)=t_{-1} \frac{1}{\epsilon}+t_{l 0} \ln \epsilon+t_{0}+t_{l 1} \epsilon \ln \epsilon+O(\epsilon)$,

$t^{\prime}\left(x^{\prime}, \theta\right)=t_{-1}^{\prime} \frac{1}{\epsilon}+t_{l 0}^{\prime} \ln \epsilon+t_{0}^{\prime}+t_{l 1}^{\prime} \epsilon \ln \epsilon+O(\epsilon)$

and the inner variables given in Eqs. (28) and (41), the scaled energy equations for the inner temperature fields in the leading orders can be written as follows

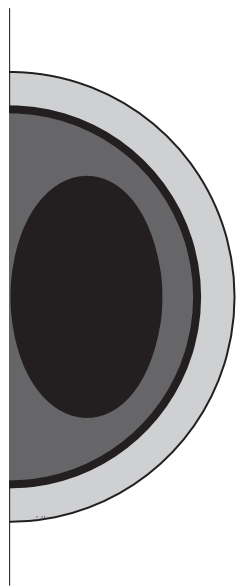

Fig. 2. A schematic diagram of the inner and outer temperature fields denoted by different colors near the surface of droplet in a coordinate system moving with the droplet velocity $V_{\infty}$. The light/dark gray and the white/black regions represent the inner $\left[t / t^{\prime} \sim 0\left(\frac{1}{\epsilon}\right) / 0\left(\frac{1}{\epsilon}\right)\right]$ and outer $\left.\left[T / T^{\prime} \sim 0(1) / 0\left(\frac{1}{\epsilon^{2}}\right)\right]\right)$ temperature fields in the continuous phase/within the droplet, respectively.

$-3 x \cos \theta \frac{\partial t_{-1}}{\partial x}+\frac{3}{2} \sin \theta \frac{\partial t_{-1}}{\partial \theta}=\frac{\partial^{2} t_{-1}}{\partial x^{2}}$,
$-3 x^{\prime} \cos \theta \frac{\partial t_{-1}^{\prime}}{\partial x^{\prime}}+\frac{3}{2} \sin \theta \frac{\partial t_{-1}^{\prime}}{\partial \theta}=\frac{\partial^{2} t_{-1}^{\prime}}{\partial x^{\prime 2}}$

The boundary conditions are

$t_{-1}(0, \theta)=t_{-1}^{\prime}(0, \theta)$,

$\delta \frac{\partial t_{-1}}{\partial x}(0, \theta)=-\frac{\partial t_{-1}^{\prime}}{\partial x^{\prime}}(0, \theta)$

$t_{-1}(x \rightarrow \infty, \theta) \rightarrow 0$,

$t_{-1}^{\prime}\left(x^{\prime} \rightarrow \infty, \theta\right) \rightarrow B+\frac{\delta}{4} x^{\prime} \sin ^{2} \theta$,

where $\delta=\sqrt{\lambda} / \beta$. We transform the independent variables from $\left[\left(x, x^{\prime}\right), \theta\right]$ to $\left[\left(\eta, \eta^{\prime}\right), \xi\right]$ and the functions from $\left(t_{-1}, t_{-1}^{\prime}\right)$ to $\left(f_{0}, f_{0}^{\prime}\right)$ as

$\left(\eta, \eta^{\prime}\right)=\left(\frac{3}{2} x \sin ^{2} \theta, \frac{3}{2} x^{\prime} \sin ^{2} \theta\right)$

$\xi=\frac{1}{2}\left(2-3 \cos \theta+\cos ^{3} \theta\right)=\frac{1}{2}(2+\cos \theta)(1-\cos \theta)^{2}$

and

$f_{0}(\eta, \xi)=t_{-1}(x, \theta)$,

$f_{0}^{\prime}\left(\eta^{\prime}, \xi\right)=t_{-1}^{\prime}\left(x^{\prime}, \theta\right)-B-\frac{\delta}{4} x^{\prime} \sin ^{2} \theta$.

The corresponding energy equations for $f_{0}, f_{0}^{\prime}$ and boundary conditions can be written as follows

$\frac{\partial f_{0}}{\partial \xi}=\frac{\partial^{2} f_{0}}{\partial \eta^{2}}$

$\frac{\partial f_{0}^{\prime}}{\partial \xi}=\frac{\partial^{2} f_{0}^{\prime}}{\partial \eta^{\prime 2}}$

and

$f_{0}(0, \xi)=f_{0}^{\prime}(0, \xi)+B$,

$\delta \frac{\partial f_{0}}{\partial \eta}(0, \xi)=-\frac{\partial f_{0}^{\prime}}{\partial \eta^{\prime}}(0, \xi)-\frac{\delta}{6}$,

$f_{0}(\eta \rightarrow \infty, \xi)=0$,

$f_{0}^{\prime}\left(\eta^{\prime} \rightarrow \infty, \xi\right)=0$ 
To solve Eq. (50), initial conditions are provided below

$f_{0}(\eta, 0)=0$,

$f_{0}^{\prime}\left(\eta^{\prime}, 0\right)=f_{0}^{\prime}\left(\eta^{\prime}, \xi(\pi)\right)=f_{0}^{\prime}\left(\eta^{\prime}, 2\right)=g_{0}\left(\eta^{\prime}\right)$,

$g_{0}\left(\eta^{\prime} \rightarrow \infty\right) \rightarrow 0$

Following the methods given by Harper and Moore [17], the solution of Eq. (50) can be obtained as

$$
\begin{aligned}
f_{0}(\eta, \xi)= & \frac{1}{1+\delta}\left\{\left(B-\frac{\delta}{6} \eta\right) \operatorname{erfc}\left(\frac{\eta}{2 \sqrt{\xi}}\right)+\frac{\delta \sqrt{\xi}}{3 \sqrt{\pi}} \exp \left(-\frac{\eta^{2}}{4 \xi}\right)\right. \\
& \left.+\frac{1}{\sqrt{\pi \xi}} \int_{0}^{\infty} g_{0}\left(\eta^{*}\right) \exp \left[-\frac{\left(\eta+\eta^{*}\right)^{2}}{4 \xi}\right] d \eta^{*}\right\}, \\
f_{0}^{\prime}\left(\eta^{\prime}, \xi\right)= & \frac{\delta}{1+\delta}\left\{-\left(B+\frac{1}{6} \eta^{\prime}\right) \operatorname{erfc}\left(\frac{\eta^{\prime}}{2 \sqrt{\xi}}\right)+\frac{\sqrt{\xi}}{3 \sqrt{\pi}} \exp \left(-\frac{\eta^{\prime 2}}{4 \xi}\right)\right\} \\
& +\frac{1}{2 \sqrt{\pi \xi}} \int_{0}^{\infty} g_{0}\left(\eta^{*}\right)\left\{\exp \left[-\frac{\left(\eta^{\prime}-\eta^{*}\right)^{2}}{4 \xi}\right]+\frac{1-\delta}{1+\delta}\right. \\
& \left.\exp \left[-\frac{\left(\eta^{\prime}+\eta^{*}\right)^{2}}{4 \xi}\right]\right\} d \eta^{*} .
\end{aligned}
$$

From Eqs. (49) and (53), we obtain the inner temperature field in its leading order near the surface of droplet

$$
\begin{aligned}
t_{-1}(0, \theta) & =f_{0}(0, \xi) \\
& =\frac{1}{1+\delta}\left[B+\frac{\delta \sqrt{\xi}}{3 \sqrt{\pi}}+\frac{1}{\sqrt{\pi \xi}} \int_{0}^{\infty} g_{0}\left(\eta^{*}\right) \exp \left(-\frac{\eta^{* 2}}{4 \xi}\right) d \eta^{*}\right] \\
& =\frac{1}{1+\delta}\left[B+\frac{\delta \sqrt{\xi}}{3 \sqrt{\pi}}+\frac{2}{\sqrt{\pi}} \int_{0}^{\infty} g_{0}\left(2 \xi^{1 / 2} \zeta\right) \exp \left(-\zeta^{2}\right) d \zeta\right] .
\end{aligned}
$$

When the inner expansion in the temperature field (43) is truncated at the zero order, we rewrite Eq. (17) as

$V_{\infty}=\frac{1}{2+3 \alpha} \int_{0}^{\pi} \sin \theta \cos \theta\left[t_{-1}(0, \theta) \frac{1}{\epsilon}+t_{l 0}(0, \theta) \ln \epsilon\right] d \theta$.

Since $\epsilon=1 / \sqrt{\mathrm{MaV}_{\infty}}$, the migration speed of the droplet is evaluated as

$V_{\infty} \approx a_{1}^{2} M a-2 a_{l 0} \ln M a+a_{0}$,

where

$a_{1}=\frac{1}{2+3 \alpha} \int_{0}^{\pi} \sin \theta \cos \theta t_{-1}(0, \theta) d \theta$

and

$a_{l 0}=\frac{1}{2+3 \alpha} \int_{0}^{\pi} \sin \theta \cos \theta t_{l 0}(0, \theta) d \theta$.

Substituting Eqs. (55) into Eq. (58), we obtain

$$
\begin{aligned}
a_{1}= & \frac{\delta}{3 \sqrt{\pi}(2+3 \alpha)(1+\delta)} \int_{0}^{\pi} \sin \theta \cos \theta \xi^{1 / 2} d \theta \\
& +\frac{2}{\sqrt{\pi}(2+3 \alpha)(1+\delta)} \int_{0}^{\pi} \sin \theta \cos \theta\left[\int_{0}^{\infty} g_{0}\left(2 \xi^{1 / 2} \zeta\right)\right. \\
& \left.\times \exp \left(-\zeta^{2}\right) d \zeta\right] d \theta .
\end{aligned}
$$

To determine the function $g_{0}$ in Eq. (60), we use the boundary condition within the droplet at the front and rear stagnation points in Eq. (52)

$$
\begin{aligned}
g_{0}\left(\eta^{\prime}\right)= & \frac{\delta}{1+\delta}\left\{-\left(B+\frac{1}{6} \eta^{\prime}\right) \operatorname{erfc}\left(\frac{\eta^{\prime}}{2 \sqrt{2}}\right)+\frac{\sqrt{2}}{3 \sqrt{\pi}} \exp \left(-\frac{\eta^{\prime 2}}{8}\right)\right\}+\frac{1}{2 \sqrt{2 \pi}} \\
& \times \int_{0}^{\infty} g_{0}\left(\eta^{*}\right)\left\{\exp \left[-\frac{\left(\eta^{\prime}-\eta^{*}\right)^{2}}{8}\right]+\frac{1-\delta}{1+\delta} \exp \left[-\frac{\left(\eta^{\prime}+\eta^{*}\right)^{2}}{8}\right]\right\} d \eta^{*} .
\end{aligned}
$$

The integral of the third term on the right-hand side of Eq. (61) is approximated as

$$
\begin{aligned}
\int_{0}^{\infty} g_{0}\left(\eta^{*}\right) h\left(\eta^{\prime}, \eta^{*}\right) d \eta^{*}= & \int_{0}^{\eta_{l}^{*}} g_{0}\left(\eta^{*}\right) h\left(\eta^{\prime}, \eta^{*}\right) d \eta^{*}+g_{0}\left(\eta_{l}^{*}\right) \\
& \times \int_{\eta_{l}^{*}}^{\infty} h\left(\eta^{\prime}, \eta^{*}\right) d \eta^{*}
\end{aligned}
$$

Then, Eq. (61) is evaluated in a linear system of equations $g_{0}\left(\eta^{\prime}\right.$

$$
\begin{aligned}
& -\frac{1}{4 \sqrt{2 \pi}} g_{0}\left(\eta_{1}^{*}\right)\left\{\exp \left[-\frac{\left(\eta^{\prime}-\eta_{1}^{*}\right)^{2}}{8}\right]+\frac{1-\delta}{1+\delta} \exp \left[-\frac{\left(\eta^{\prime}+\eta_{1}^{*}\right)^{2}}{8}\right]\right\} \Delta \eta^{*} \\
& -\frac{1}{4 \sqrt{2 \pi}} g_{0}\left(\eta_{N+1}^{*}\right)\left\{\exp \left[-\frac{\left(\eta^{\prime}-\eta_{N+1}^{*}\right)^{2}}{8}\right]+\frac{1-\delta}{1+\delta} \exp \left[-\frac{\left(\eta^{\prime}+\eta_{N+1}^{*}\right)^{2}}{8}\right]\right\} \Delta \eta^{*} \\
& -\frac{1}{2 \sqrt{2 \pi}} \sum_{j=2}^{N} g_{0}\left(\eta_{j}^{*}\right)\left\{\exp \left[-\frac{\left(\eta^{\prime}-\eta_{j}^{*}\right)^{2}}{8}\right]+\frac{1-\delta}{1+\delta} \exp \left[-\frac{\left(\eta^{\prime}+\eta_{j}^{*}\right)^{2}}{8}\right]\right\} \Delta \eta^{*} \\
& -\frac{1}{2} g_{0}\left(\eta_{N+1}^{*}\right)\left[\operatorname{erfc}\left(\frac{\eta_{N+1}^{*}+\eta^{\prime}}{2 \sqrt{2}}\right)+\frac{1-\delta}{1+\delta} \operatorname{erfc}\left(\frac{\eta_{N+1}^{*}+\eta^{\prime}}{2 \sqrt{2}}\right)\right] \\
& =\frac{\delta}{1+\delta}\left[-\left(B+\frac{1}{6} \eta^{\prime}\right) \operatorname{erfc}\left(\frac{\eta^{\prime}}{2 \sqrt{2}}\right)+\frac{\sqrt{2}}{3 \sqrt{\pi}} \exp \left(-\frac{\eta^{\prime 2}}{8}\right)\right],
\end{aligned}
$$

where $\eta_{N+1}^{*}=\eta_{l}^{*}$ and $\Delta \eta^{*}=\eta_{l}^{*} / N$. The physical coefficients used in the space experiment [12] with the uniform temperature gradient $G=12 \mathrm{~K} / \mathrm{cm}$ for the continuous phase of Fluorinert FC-75 and the droplet of 5 cst silicon oil at $T=333 \mathrm{~K}$ are adopted here to yield $\alpha=0.342, \beta=0.571$ and $\lambda=0.299$. A typical value for $\eta_{l}^{*}$ is chosen as 3. Using the trial and error method to satisfy the above approximation, we determine the unknown constant $B=0.1935$ and obtain the dependence of $g_{0}$ on $\eta^{\prime}$ as shown in Fig. 3. From Eq. (60), we can determine the root-mean-square of the leading order term of the migration speed as

$a_{1}=1.15 \times 10^{-2}$.

Although equations and boundary conditions describing the second order term of the migration speed can be obtained, we are unable to find an analytical result for $t_{10}$ in Eq. (56). Under the truncation after the leading order term in Eq. (57), we obtain the migration speed of the droplet

$V_{\infty} \approx 1.3 \times 10^{-4} \mathrm{Ma}$.

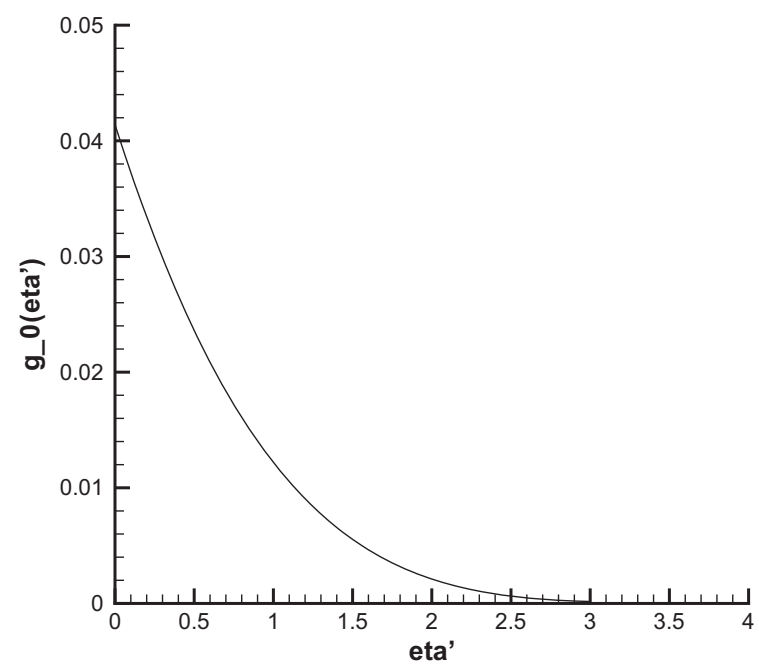

Fig. 3. Function $g_{0}$ versus $\eta^{\prime}$ determined from Eq. (63). 
By using the migration speed $V_{\infty}$, the adding thermal source $\Omega$ in Eq. (22) can be determined as

$\Omega=G v_{0} V_{\infty}\left(1+\frac{\lambda}{2 \beta}\right) \approx 0.1 \mathrm{Ma} \mathrm{K} / \mathrm{s}$

\section{Conclusions and discussion}

In this paper, the conservative integral thermal flux across the surface for a steady thermocapillary droplet migration in a uniform temperature gradient at large Re and Ma numbers has been guaranteed by adding a thermal source in the droplet. Under the assumptions of quasi-steady state and non-deformation of the droplet, we have made an analytical result for the steady thermocapillary migration of droplet with the thermal source at large Re and Ma numbers. The result shows that the thermocapillary droplet migration speed slowly increases with the increase of Ma number.

We emphasize that the modeling of the adding thermal source in the droplet is used to investigate the effect of integral thermal flux across the surface on the thermocapillary droplet migration process. It is one of the physical means to satisfy the conservative integral thermal flux boundary condition in the steady migration process at large Ma numbers[15]. On the one hand, in general, when the droplet moves forward under the uniform temperature gradient, the thermal energy in the droplet is imported from the upper surface of the droplet and exported from the lower surface. The adding thermal source in the droplet can decrease/increase the inner thermal flux from the interface/inside to the inside/interface at the upper/lower surface of the droplet and satisfy the thermal flux boundary condition at the surface of droplet for the steady migration process. On the other hand, at large Ma numbers, the heat advection around the droplet is a more significant mechanism for heat transfer across/around the droplet. It is always keeping the weak transport of thermal energy from outside of the droplet to inside. Thus, the adding thermal source is conducive to providing the thermal energy in the droplet to meet the requirement put forward by the quasi-steady migration process. Meanwhile, the heat transport across the droplet surface becomes weaker than the heat convection as Ma number increases. To reach the steady migration process, more thermal energy in the droplet will be provided. The proportional relationship of the thermal source to Ma number in Eq. (66) qualitatively confirms the above requirement of more thermal energy in the droplet.

In final, some suggestions are provided in order that the thermal source in the droplet can be implementend in a real experiment. As given in Eqs. (1) and (66) and shown in Fig. 1, the thermal source depending on Ma number will be added in the droplet when it starts to move under the uniform temperature gradient. The heat radiant technology may be one of the possible physical means to add the thermal source in the droplet.

\section{Conflict of Interest}

None declared.

\section{Acknowledgments}

We thank the National Science Foundation for partial support through the Grant No. 11172310 and the IMECH/SCCAS SHENTENG $1800 / 7000$ research computing facilities for assisting in the computation.

\section{References}

[1] R.S. Subramanian, R. Balasubramaniam, The Motion of Bubbles and Drops in Reduced Gravity, Cambridge University Press, Cambridge, England, 2001.

[2] A.A. Darhuber, S.M. Troian, Principles of microfluidic actuation by modulation of surface stresses, Annu. Rev. Fluid Mech. 37 (2005) 425.

[3] N.O. Young, J.S. Goldstein, M.J. Block, The motion of bubbles in a vertical temperature gradient, J. Fluid Mech. 6 (1959) 350.

[4] R.S. Subramanian, Slow migration of a gas bubble in a thermal gradient, AIChE J. 27 (1981) 646.

[5] R. Balasubramaniam, A.-T. Chai, Thermocapillary migration of droplet: an exact solution for small Marangoni numbers, J. Colloid Interface Sci. 119 (1987) 531.

[6] B. Braun, C. Ikier, H. Klein, Thermocapillary migration of droplet in a binary mixture with miscibility gap during liquid/liquid phase separation under reduced gravity, J. Colloid Interface Sci. 159 (1993) 515.

[7] M.K. Smith, Thermocapillary migration of a two-dimensional liquid droplet on a solid surface, J. Fluid Mech. 294 (1995) 209.

[8] J.Z. Chen, S.M. Troian, A.A. Darhuber, S. Wagner, Effect of contact angle hysteresis on thermocapillary droplet actuation, J. Appl. Phys. 97 (2005) 014906.

[9] C. Song, K. Kim, K. Lee, H.K. Pak, Thermocapillary control of oil droplet motion on a solid substrate, Appl. Phys. Lett. 93 (2008) 084102.

[10] H.-B. Nguyen, J.-C. Chen, A numerical study of thermocapillary migration of a small liquid droplet on a horizontal solid surface, Phys. Fluids 22 (2012) 062102.

[11] R. Balasubramaniam, R.S. Subramanian, The migration of a droplet in a uniform temperature gradient at large Marangoni numbers, Phys. Fluids 12 (2000) 733.

[12] X. Ma, R. Balasubramiam, R.S. Subramanian, Numerical simulation of thermocapillary droplet motion with internal circulation, Numer. Heat Transfer A35 (1999) 291.

[13] P.H. Hadland, R. Balasubramaniam, G. Wozniak, Thermocapillary migration of bubbles and droplets at moderate to large Marangoni number and moderate Reynolds number in reduced gravity, Exper. Fluid 26 (1999) 240.

[14] J.C. Xie, H. Lin, P. Zhang, F. Liu, W.R. Hu, Experimental investigation on thermocapillary droplet migration at large Marangoni number in reduced gravity, J. Colloid Interface Sci. 285 (2005) 737.

[15] Z.-B. Wu, W.-R. Hu, Effects of Marangoni numbers on thermocapillary droplet migration: constant for quasi-steady state?, J Math. Phys. 54 (2013) 023102.

[16] Z.-B. Wu, W.-R. Hu, Thermocapillary migration of a planar droplet at moderate and large Marangoni numbers, Acta Mech. 223 (2012) 609.

[17] J.F. Harper, D.W. Moore, The motion of a spherical liquid drop at high Reynolds number, J. Fluid Mech. 32 (1968) 367.

[18] A. Crespo, J. Jimenez-Fernandez, Thermocapillary migration of bubbles at moderately large Reynolds numbers, in: H.J. Rath (Ed.), Microvity Fluid Mechanics, Springer-Verlag, 1992, pp. 405-511.

[19] R. Balasubramaniam, R.S. Subramanian, Thermocapillary bubble migrationthermal boundary layers for large Marangoni numbers, Int. J. Multiphase Flow 22 (1996) 593. 